\title{
Primeiro registro documentado de Leucopternis melanops (Latham, 1790) (Aves: Accipitridae) no estado do Maranhão e atualização da distribuição geográfica da espécie no Brasil \\ First documented record of Leucopternis melanops (Latham, 1790) (Aves: Accipitridae) in the state of Maranhão and update of the geographic distribution of this species in Brazil
}

\author{
Leonardo Victor Soares Pinheiro (1) | Pablo Vieira Cerqueira ${ }^{\text {III }}$ |l| (D) | Gabriel Augusto Leite ${ }^{\text {IV }}$ (1) \\ Marcos de Souza Fialhov ${ }^{\vee}$ | Gustavo Gonsioroskiv ${ }^{\text {(DI }}$ \\ Instituto Ecos de Gaia. São Luís, Maranhão, Brasil
}

"Museu Paraense Emílio Goeldi/Universidade Federal do Pará. Programa de Pós-Graduação em Zoologia.

Laboratório de Ornitologia e Biologia Molecular. Belém, Pará, Brasil

IIIPinima Birding - Nature Tours. Belém, Pará, Brasil

IVInstituto Nacional de Pesquisas da Amazônia. Programa de Pós-Graduação em Genética, Conservação e Biologia Evolutiva.

Manaus, Amazonas, Brasil

${ }^{\vee}$ Centro Nacional de Pesquisa e Conservação de Aves Silvestres - CEMAVE. Cabedelo, Paraíba, Brasil

v'Universidade Estadual do Maranhão. Programa de Pós-Graduação em Biodiversidade, Ambiente e Saúde. Caxias, Maranhão, Brasil

\begin{abstract}
Resumo: O gavião-de-cara-preta, Leucopternis melanops (Latham, 1790), é o representante do gênero com a maior distribuição geográfica, sendo regular na calha norte do rio Amazonas e com raros registros na calha sul (Acre, Amazonas, Mato Grosso, Pará e Rondônia). Em 24 de junho de 2018, um indivíduo de L. melanops foi encontrado no município de Centro Novo do Maranhão, estado do Maranhão, Brasil, dentro dos limites da Reserva Biológica do Gurupi, sendo este o primeiro registro documentado para o estado. Este registro, somado a outros, serviu para atualizar os limites da distribuição da espécie no Brasil, além de destacar a importância do mosaico Gurupi em manter espécies pouco comuns.
\end{abstract}

Palavras-chave: Reserva Biológica do Gurupi. Unidades de conservação. Área de endemismo Belém. Limites de distribuição.

\begin{abstract}
The Black-faced hawk, Leucopternis melanops (Latham, 1790), is the representative of the genus with the greatest geographic distribution, being common on the northern bank of the Amazon River and with rare records on the southern bank (Acre, Amazonas, Mato Grosso, Pará and Rondônia). On June 24, 2018, an individual of L. melanops was found in the municipality of Centro Novo do Maranhão, state of Maranhão, Brazil, within the limits of the Gurupi Biological Reserve, which is the first documented record for the state. This record, added to others, served to update the limits of the distribution of $L$. melanops in Brazil, in addition to highlighting the importance of the Gurupi mosaic in maintaining unusual species.
\end{abstract}

Keyword: Biological Reserve of Gurupi. Conservation units. Belém area of endemism. Distribution range limits.

PINHEIRO, L. V. S., P. V. CERQUEIRA, G. A. LEITE, M. S. FIALHO \& G. GONSIOROSKI, 2021. Primeiro registro documentado de Leucopternis melanops (Latham, 1790) (Aves: Accipitridae) no estado do Maranhão e atualização da distribuição geográfica da espécie no Brasil. Boletim do Museu Paraense Emílio Goeldi. Ciências Naturais 16(1): 131-143. DOI: http://doi.org/10.46357/bcnaturais.v16i1.450. Autor para correspondência: Leonardo Victor Soares Pinheiro. Travessa das Flores, s/n - Trizidela. São Benedito do Rio Preto, MA, Brasil. CEP65440-000 (leovictorsp13@gmail.com).

Recebido em 03/12/2020

Aprovado em 02/03/2021

Responsabilidade editorial: Adriano Oliveira Maciel
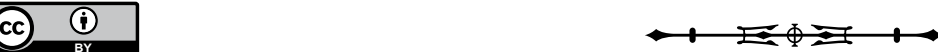
O gênero Leucopternis, componente da família Accipitridae, possui três espécies, Leucopternis semiplumbeus Lawrence, 1861, L. kuhli Bonaparte, 1850 e L. melanops (Latham, 1790), todas de hábitos florestais (Bierregaard et al., 2020a, 2020b, 2020c). Leucopternis semiplumbeus distribui-se do norte do Equador até o norte de Honduras (Bierregaard et al., 2020b). Por sua vez, L. kuhli ocorre no noroeste da Bolívia, no Peru (Madre de Dios), no Equador e no Brasil, na margem sul do rio Amazonas (Barlow et al., 2002; Amaral et al., 2007; Portes et al., 2011; Lees et al., 2012; Bierregaard et al., 2020c). Enquanto L. melanops é o representante com maior distribuição, presente em Peru (Madre de Dios e Loreto), no Equador, na Colômbia, na Venezuela, nas Guianas, apresentando ampla distribuição na Amazônia brasileira. Contudo, é mais comumente encontrado na calha norte do rio Amazonas (Barlow et al., 2002; Amaral et al., 2007; Shrum et al., 2011; Bierregaard et al., 2020a).

O primeiro registro de L. melanops no Brasil foi realizado por Johann Natterer, que coletou dois exemplares em Barra do Rio Negro, hoje Manaus, em expedição executada entre 1817 e 1835 (Pelzeln, 1871). Cerca de um século depois, em 1921, outro indivíduo foi coletado no município de Óbidos, ao norte do rio Amazonas, no estado do Pará, por S. M. Klages (CM-Birds, 2020). Em 1931, um espécime de L. melanops foi coletado por A. M. Olalla, em Tauari, baixo Tapajós (Amadon, 1964). Este foi o primeiro registro documentado de L. melanops na margem sul do rio Amazonas (Amaral et al., 2007). A partir deste registro, outros indivíduos foram documentados nos estados do Acre (Amaral et al., 2007), Amazonas (Amaral et al., 2007; Bierregaard et al., 2020a), Mato Grosso (Borges, 2016; Bierregaard et al., 2020a), Pará (Amaral et al., 2007; Moura, 2012; Bierregaard et al., 2020a) e Rondônia (Roth, 2011; Bierregaard et al., 2020a).

Registros da espécie ao sul do rio Amazonas são relativamente incomuns, especialmente no extremo leste na região biogeográfica conhecida como Centro de Endemismo Belém (CEB) (Da Silva et al., 2005).
Nessa região, foram feitos apenas dois registros: o primeiro deles foi realizado por Emílio Dente, em 1959, na região do rio Capim, nas imediações da antiga BR-14, atual BR-010 (Amaral et al., 2007). O segundo também ocorreu no rio Capim, no município de Goianésia do Pará, quando um indivíduo foi fotografado por Alexander Lees, Nárgila Moura e Ian Thompson, em 2013 (Lees, 2013).

Em 24 de junho de 2018, pela manhã, durante a implantação da segunda campanha do Protocolo Avançado de Monitoramento de Aves, que faz parte do Programa Nacional de Monitoramento da Biodiversidade - Programa Monitora (Bispo et al., 2016; Ribeiro, 2018), na Reserva Biológica do Gurupi (REBIO Gurupi), estado do Maranhão, L.V.S.P e P.V.C. observaram e fotografaram um indivíduo de $L$. melanops na trilha B $\left(03^{\circ} 15^{\prime}\right.$ 07,45" S; $46^{\circ} 45^{\prime}$ 08,80" O; Figura 1). O gavião foi avistado em vegetação de floresta ombrófila de terra firme com algumas clareiras antigas. $O$ indivíduo apresentava as partes inferiores, cabeça e pescoço brancos, com finas linhas pretas visíveis apenas na fronte próxima ao bico; dorso e asas pretas com poucas manchas brancas; a cauda também era preta, com apenas uma faixa branca visível, assim como a ponta das retrizes; máscara preta ampla e contrastante com a face branca; cera e tarsos alaranjados. Tais características indicam que o indivíduo seja um adulto e nos permitem diferenciá-lo do seu congênere e simpátrico L. kuhli (Figura 2). Portanto, este é o primeiro registro documentado para o estado, onde apenas L. kuhli havia sido documentado (Oren, 1991; Lima et al., 2014; Carvalho et al., 2020).

Adicionalmente ao registro aqui reportado, que expande em aproximadamente $200 \mathrm{~km}$ o limite leste da distribuição da espécie, compilamos os registros reportados em literatura científica (e.g. Barlow et al., 2002; Amaral et al., 2007) e nas plataformas digitais Gbif, Wikiaves e Xenocanto até outubro de 2020 (Apêndice) e disponibilizamos uma proposta da abrangência da distribuição da espécie, com sugestões de atualização da distribuição geográfica dela (Figura 1). 


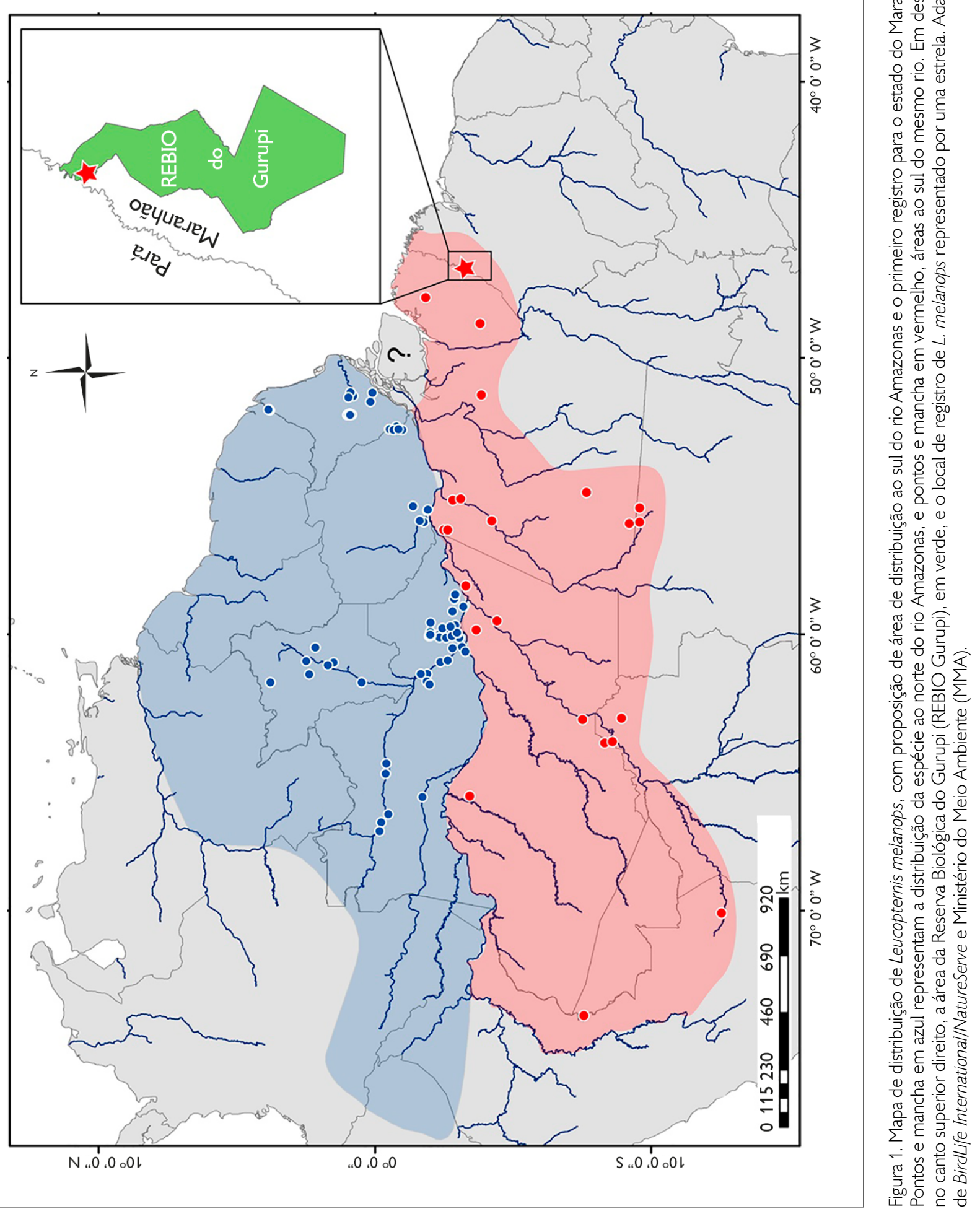




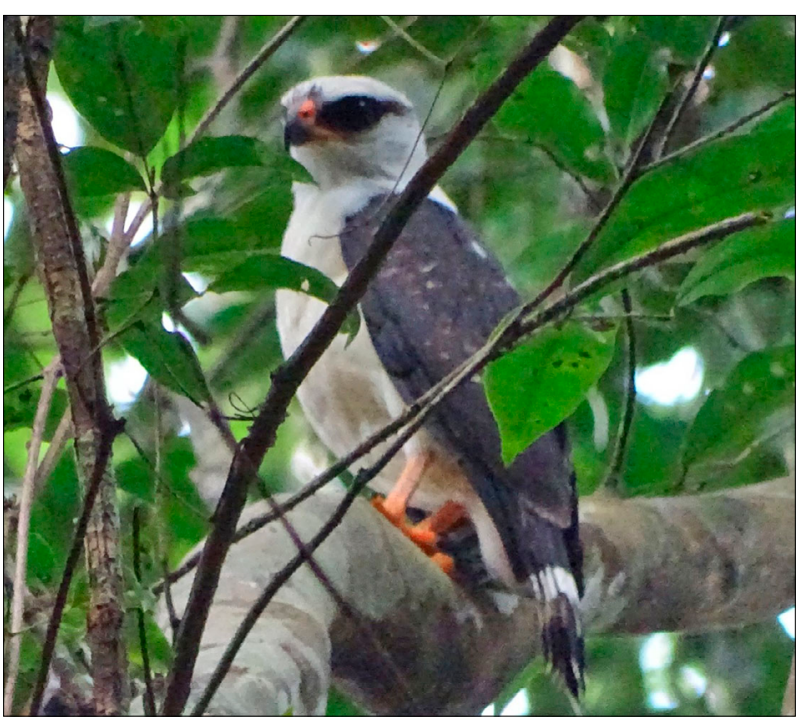

Figura 2. Indivíduo de Leucopternis melanops registrado durante aplicação de Protocolo Avançado de Monitoramento de Aves na REBIO Gurupi. Foto: Leonardo Victor (2018).

Por fim, esse registro, juntamente a outros táxons documentados (Oren, 1991; Lima \& Raices, 2012; Lima et al., 2014; Carvalho et al., 2017; Alteff et al., 2019; Carvalho et al., 2020; Moraes et al., 2020), mostra a importância do mosaico Gurupi para a conservação biológica do CEB, onde estão inseridas as terras indígenas Alto do Turiaçu, Awá e Caru e a REBIO Gurupi, indicando a necessidade de mais esforços para inventariar a biodiversidade e medidas que ajudem na manutenção da integridade ambiental dessas áreas.

\section{AGRADECIMENTOS}

Agradecemos ao Programa Monitora, ao Centro Nacional de Pesquisa e Conservação de Aves Brasileiras (CEMAVE) e à Reserva Biológica do Gurupi (REBIO Gurupi), por promoverem as expedições do Protocolo Avançado de Monitoramento de Comunidades de Aves em Unidades de Conservação Federais na REBIO Gurupi. Ao Programa de Áreas Protegidas da Amazônia (Programa ARPA), por ter fornecido recursos para aplicação do protocolo. Aos dois revisores anônimos, pelas importantes sugestões. E, por fim, agradecemos a Guy M. Kirwan, por ajustes na tradução.

\section{REFERÊNCIAS}

ALTEFF, E. F., G. GONSIOROSKI, M. BARREIROS, L. G. C. D. O. TORRES, A. R. CAMILO, H. B. MOZERLE, A. E. B. A. D. SOUSA, C. A. B. MEDOLAGO, C. MARTÍNEZ, D. M. LIMA \& F. K. UBAID, 2019. The rarest of the rare: rediscovery and status of the critically endangered Belem Curassow, Crax fasciolata pinima (Pelzeln, 1870). Papéis Avulsos de Zoologia 59: e20195946. DOI: http://dx.doi.org/10.11606/1807-0205/2019.59.46

AMADON, D., 1964. Taxonomic notes on birds of prey. American Museum Novitates 2166: 1-24.

AMARAL, F. S. R., L. F. SILVEIRA \& B. M. WHITNEY, 2007. New localities for the Black-faced Hawk (Leucopternis melanops) south of the Amazon River and description of the immature plumage of the White-browed Hawk (Leucopternis kuhli). The Wilson Journal of Ornithology 119(3): 450-454

BARLOW, J., T. HAUGAASEN \& C. A. PERES, 2002. Sympatry of Black-faced Leucopternis melanops and White-browed Hawks L. kuhli along the lower rio Tapajós, Pará, Brazil. Cotinga 18: 77-79.

BIERREGAARD, R. O., P. F. D. BOESMAN \&J. S. MARKS, 2020a. Black-faced Hawk (Leucopternis melanops), version 1.0. In: J. DEL HOYO, A. ELLIOTT, J. SARGATAL, D. A. CHRISTIE \& E. DE JUANA (Ed.): Birds of the World. Cornell Lab of Ornithology, Ithaca. DOI: https://doi.org/10.2173/bow.blfhaw1.01

BIERREGAARD, R. O., P. F. D. BOESMAN \&J. S. MARKS, 2020b. Semiplumbeous Hawk (Leucopternis semiplumbeus), version 1.0. In: J. DEL HOYO, A. ELLIOTT, J. SARGATAL, D. A. CHRISTIE \& E. DE JUANA (Ed.): Birds of the World Cornell Lab of Ornithology. Cornell Lab of Ornithology, Ithaca. DOI: https://doi.org/10.2173/ bow.semhaw.01

BIERREGAARD, R. O., P. F. D. BOESMAN \& J. S. MARKS, 2020c. White-browed Hawk (Leucopternis kuhli), version 1.0. In In: J. DEL HOYO, A. ELLIOTT, J. SARGATAL, D. A. CHRISTIE \& E. DE JUANA (Ed.): Birds of the World Cornell Lab of Ornithology. Cornell Lab of Ornithology, Ithaca. DOI: https://doi.org/10.2173/ bow.whbhaw2.01

BISPO, A. Â., A. G. AGUIAR, N. R. ALMEIDA, C. G. MACHADO, M. COHN-HAFT, P. F. DEVELEY, T. O. LARANJEIRAS, A. L. CAROLINA \& M. UEHARA-PRADO, 2016. Protocolo para monitoramento de comunidades de aves em Unidades de Conservação Federais. Biodiversidade Brasileira-BioBrasil (1): 153-173.

BORGES, C. A., 2016. WA2103230, Leucopternis melanops (Latham, 1790). Wiki Aves - A Enciclopédia das Aves do Brasil. Disponível em: http://www.wikiaves.com/2103230. Acesso em: 24 outubro 2020.

CARNEGIE MUSEUM BIRDS COLLECTION (CM-BIRDS), 2020. Leucopternis melanops CMNH-P83029. SpeciesLink. Disponível em: http://www.splink.org.br. Acesso em: 31 Agosto de 2020.

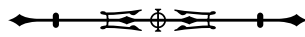


CARVALHO, D. L., T. SOUSA-NEVES, P. V. CERQUEIRA, G. GONSIOROSKI, S. M. SILVA, D. P. SILVA \& M. P. D. SANTOS, 2017. Delimiting priority areas for the conservation of endemic and threatened Neotropical birds using a nichebased gap analysis. PLoS One 12(2): e0171838. DOI: https://doi.org/10.1371/journal. pone.0171838

CARVALHO, D. L., S. M. SILVA, T. SOUSA-NEVES, D. P. SILVA \& M. P. D. SANTOS, 2020. An updated documented inventory and new records of bird species for the Brazilian state of Maranhão. Ornithology Research 28(2): 77-85. DOI: https://doi.org/10.1007/ s43388-020-00013-2

DA SILVA, J. M. C., A. B. RYLANDS \& G. A. B. DA FONSECA, 2005. The fate of the Amazonian areas of endemism. Conservation Biology 19(3): 689-694.

LEES, A. C., N. G. DE MOURA, A. SANTANA, A. ALEIXO, J. BARLOW, E. BERENGUER, J. FERREIRA \& T. A. GARDNER, 2012. Paragominas: a quantitative baseline inventory of an eastern Amazonian avifauna. Revista Brasileira de Ornitologia 20(48): 26.

LEES, A. C., 2013. [WA1162088, Leucopternis melanops (Latham, 1790)]. Wiki Aves - A Enciclopédia das Aves do Brasil. Disponível em: http://www.wikiaves.com/1162088. Acesso em: 31 agosto 2020.

LIMA, D. M., C. MARTÍNEZ \& D. S. L. RAÍCES, 2014. An avifaunal inventory and conservation prospects for the Gurupi Biological Reserve, Maranhão, Brazil. Revista Brasileira de Ornitologia 22(4): 317-340.

LIMA, D. M. \& D. S. L. RAICES, 2012. Primeiro registro de Psophia obscura Pelzeln, 1857 e Dendrocincla merula badia Zimmer, 1934 para a Reserva Biológica do Gurupi, Maranhão, Brasil. Ornithologia 5(1): 39-42.

MORAES, K. F., M. P. D. SANTOS, G. S. R. GONÇALVES, G. L. OLIVEIRA, L. B. GOMES \& M. G. M. LIMA, 2020. Climate change and bird extinctions in the Amazon. Plos One 15(7): e0236103. DOI: https://doi.org/10.1371/journal.pone.0236103
MOURA, T., 2012. WA839366, Leucopternis melanops (Latham, 1790). Wiki Aves - A Enciclopédia das Aves do Brasil. Disponível em: http://www.wikiaves.com.br/839366. Acesso em: 24 outubro 2020.

OREN, D. C., 1991. Aves do estado do Maranhão. Goeldiana. Zoologia 9: 1-55.

PELZELN, A. V., 1871. Zur Ornithologie brasiliens: Resultate von Johann Natterers Reisen in den Jahren 1817 bis 1835. A. Pichler's Witwe \& Sohn (Itinerarium von Natterer's Reisen in Brasilien 1817 1835), Viena. Disponível em: https://www.biodiversitylibrary.org/ item/21811\# page/14/mode/1up. Acesso em: 27 janeiro 2021.

PORTES, C. E. B., L. S. CARNEIRO, F. SCHUNCK, M. S. S. SILVA, K. J. ZIMMER, A. WHITTAKER, F. POLETTO, L. F. SILVEIRA \& A. ALEIXO, 2011. Annotated checklist of birds recorded between 1998 and 2009 at nine areas in the Belém area of endemism, with notes on some range extensions and the conservation status of endangered species. Revista Brasileira de Ornitologia 19(2): 167-184.

RIBEIRO, K. T., 2018. Estratégia do Programa Nacional de Monitoramento da Biodiversidade - Programa Monitora: estrutura, articulações, perspectivas. ICMBio, Brasília. Disponível em: https://www.icmbio.gov.br/portal/images/stories/o-que-fazemos/ monitoramento/Programa_Monitora_-_Estrat\%C3\%A9gia_Geral. pdf. Acesso em: 24 outubro 2020.

ROTH, P. R., 2011. WA422050, Leucopternis melanops (Latham, 1790). Wiki Aves - A Enciclopédia das Aves do Brasil. Disponível em: http://www.wikiaves.com.br/422050\&tm $=f \& t=s \& s=10215 \&$ $\mathrm{o}=\mathrm{mp} \& \mathrm{o}=\mathrm{mp}$. Acesso em: 24 outubro 2020 .

SHRUM, P. L., W. W. BOWERMAN, D. G. OLAECHEA \& R. AMABLE, 2011. More records of sympatry of black-faced hawk (Leucopternis melanops) and white-browed hawk (L. kuhli) in Madre de Dios, Peru. Journal of Raptor Research 45(1): 104-105. DOI: https://doi.org/10.3356/JRR-10-47.1 
Apêndice. Dados complementares levantados para elaboração da nota. Fontes: American Museum of Natural History (AMNH), Academy of Natural Sciences of Drexel University (ANSP), Cleveland Museum of Natural History (CMNH), EBIRD, Field Museum Natural History (FMNH), Inaturalist (IN), Instituto Nacional de Pesquisas da Amazônia (INPA), Macaulay Library (ML), Museu Nacional do Rio de Janeiro (MNRJ), Museu Paraense Emílio Goeldi (MPEG), Museu de Zoologia da Universidade de São Paulo (MZUSP), Smithsonian National Museum of Natural History (NMNH), Wikiaves (WA), Xeno-canto (XC). Tombo: código dos museus e plataformas digitais utilizadas na pesquisa.

(Continua)

\begin{tabular}{|c|c|c|c|}
\hline Estado & Fonte & Tombo & Ano \\
\hline Acre & MPEG & 52705 & 1966 \\
\hline Amapá & MPEG & 17251 & 1959 \\
\hline Amapá & MPEG & 47656 & 1965 \\
\hline Amapá & MPEG & 24833 & 1965 \\
\hline Amapá & MZUSP & 62646 & 1965 \\
\hline Amapá & MPEG & 53428 & 1997 \\
\hline Amapá & WA & 1433708 & 2000 \\
\hline Amapá & $x C$ & 85200 & 2000 \\
\hline Amapá & EBIRD & 609798168 & 2009 \\
\hline Amapá & WA & 148954 & 2009 \\
\hline Amapá & WA & 748233 & 2012 \\
\hline Amapá & WA & 1540505 & 2012 \\
\hline Amapá & WA & 2713927 & 2013 \\
\hline Amapá & WA & 2430466 & 2013 \\
\hline Amapá & EBIRD & 438523785 & 2014 \\
\hline Amapá & EBIRD & 361990595 & 2015 \\
\hline Amapá & EBIRD & 361994268 & 2015 \\
\hline Amapá & EBIRD & 362005542 & 2015 \\
\hline Amapá & EBIRD & 361999359 & 2015 \\
\hline Amapá & WA & 2367457 & 2015 \\
\hline Amapá & EBIRD & 436653216 & 2016 \\
\hline Amapá & EBIRD & 385153574 & 2016 \\
\hline Amapá & EBIRD & 377807800 & 2016 \\
\hline Amapá & EBIRD & 377585363 & 2016 \\
\hline Amapá & WA & 2327248 & 2016 \\
\hline Amapá & WA & 2512133 & 2016 \\
\hline Amapá & EBIRD & 480075114 & 2017 \\
\hline Amapá & EBIRD & 465690002 & 2017 \\
\hline Amapá & WA & 3045250 & 2017 \\
\hline Amapá & EBIRD & 684565418 & 2018 \\
\hline Amapá & EBIRD & 684538376 & 2018 \\
\hline Amapá & EBIRD & 669848448 & 2018 \\
\hline Amapá & EBIRD & 578896638 & 2018 \\
\hline
\end{tabular}


Apêndice.

\begin{tabular}{|c|c|c|c|}
\hline Estado & Fonte & Tombo & Ano \\
\hline Amapá & EBIRD & 570976540 & 2018 \\
\hline Amapá & WA & 3265086 & 2018 \\
\hline Amapá & WA & 3813453 & 2019 \\
\hline Amapá & WA & 3451025 & 2019 \\
\hline Amapá & $\mathrm{FMNH}$ & 391235 & \\
\hline Amazonas & $\mathrm{NMNH}$ & 316397 & 1930 \\
\hline Amazonas & $\mathrm{FMNH}$ & 100356 & 1932 \\
\hline Amazonas & $\mathrm{FMNH}$ & 101512 & 1937 \\
\hline Amazonas & EBIRD & 332843375 & 1995 \\
\hline Amazonas & EBIRD & 198698726 & 1997 \\
\hline Amazonas & $M L$ & 117250 & 1997 \\
\hline Amazonas & WA & 87627 & 2004 \\
\hline Amazonas & $X C$ & 10127 & 2006 \\
\hline Amazonas & EBIRD & 482653094 & 2007 \\
\hline Amazonas & EBIRD & 460434262 & 2007 \\
\hline Amazonas & EBIRD & 83101032 & 2007 \\
\hline Amazonas & $\mathrm{FMNH}$ & 456425 & 2007 \\
\hline Amazonas & MPEG & 0062387 & 2007 \\
\hline Amazonas & EBIRD & 526548036 & 2008 \\
\hline Amazonas & EBIRD & 526669935 & 2008 \\
\hline Amazonas & EBIRD & 525583618 & 2008 \\
\hline Amazonas & EBIRD & 643195928 & 2009 \\
\hline Amazonas & EBIRD & 529289959 & 2009 \\
\hline Amazonas & EBIRD & 127036038 & 2011 \\
\hline Amazonas & $X C$ & 113627 & 2011 \\
\hline Amazonas & EBIRD & 458023062 & 2012 \\
\hline Amazonas & EBIRD & 197112415 & 2012 \\
\hline Amazonas & EBIRD & 166870115 & 2012 \\
\hline Amazonas & INPA & 4622 & 2012 \\
\hline Amazonas & WA & 737009 & 2012 \\
\hline Amazonas & WA & 740548 & 2012 \\
\hline Amazonas & $X C$ & 118475 & 2012 \\
\hline Amazonas & EBIRD & 202082732 & 2013 \\
\hline Amazonas & WA & 1337230 & 2013 \\
\hline Amazonas & WA & 1215039 & 2013 \\
\hline Amazonas & WA & 1267156 & 2013 \\
\hline Amazonas & $X C$ & 315413 & 2013 \\
\hline
\end{tabular}


Apêndice.

\begin{tabular}{|c|c|c|c|}
\hline Estado & Fonte & Tombo & Ano \\
\hline Amazonas & EBIRD & 300584482 & 2014 \\
\hline Amazonas & EBIRD & 270910901 & 2014 \\
\hline Amazonas & EBIRD & 269922774 & 2014 \\
\hline Amazonas & EBIRD & 269922730 & 2014 \\
\hline Amazonas & EBIRD & 268977943 & 2014 \\
\hline Amazonas & EBIRD & 279330158 & 2014 \\
\hline Amazonas & $X C$ & 188622 & 2014 \\
\hline Amazonas & EBIRD & 566668225 & 2015 \\
\hline Amazonas & EBIRD & 454384778 & 2015 \\
\hline Amazonas & EBIRD & 445787351 & 2015 \\
\hline Amazonas & EBIRD & 442696713 & 2015 \\
\hline Amazonas & EBIRD & 412677460 & 2015 \\
\hline Amazonas & EBIRD & 381690039 & 2015 \\
\hline Amazonas & EBIRD & 370303504 & 2015 \\
\hline Amazonas & EBIRD & 351336671 & 2015 \\
\hline Amazonas & EBIRD & 350472843 & 2015 \\
\hline Amazonas & EBIRD & 346592309 & 2015 \\
\hline Amazonas & EBIRD & 345147002 & 2015 \\
\hline Amazonas & EBIRD & 343545767 & 2015 \\
\hline Amazonas & EBIRD & 336002887 & 2015 \\
\hline Amazonas & EBIRD & 335806543 & 2015 \\
\hline Amazonas & EBIRD & 335762762 & 2015 \\
\hline Amazonas & EBIRD & 335142311 & 2015 \\
\hline Amazonas & EBIRD & 334320359 & 2015 \\
\hline Amazonas & EBIRD & 334025332 & 2015 \\
\hline Amazonas & EBIRD & 334012132 & 2015 \\
\hline Amazonas & EBIRD & 334105569 & 2015 \\
\hline Amazonas & EBIRD & 333810352 & 2015 \\
\hline Amazonas & EBIRD & 333607862 & 2015 \\
\hline Amazonas & EBIRD & 333181704 & 2015 \\
\hline Amazonas & EBIRD & 332767172 & 2015 \\
\hline Amazonas & EBIRD & 332678495 & 2015 \\
\hline Amazonas & EBIRD & 332641369 & 2015 \\
\hline Amazonas & EBIRD & 332526598 & 2015 \\
\hline Amazonas & EBIRD & 330671304 & 2015 \\
\hline Amazonas & EBIRD & 330728650 & 2015 \\
\hline Amazonas & EBIRD & 315192957 & 2015 \\
\hline
\end{tabular}


Apêndice.

\begin{tabular}{|c|c|c|c|}
\hline & & & \\
\hline Lstauv & & गणा100 & काषि \\
\hline Amazonas & EBIRD & 314344489 & 2015 \\
\hline Amazonas & EBIRD & 334981408 & 2015 \\
\hline Amazonas & EBIRD & 334270468 & 2015 \\
\hline Amazonas & EBIRD & 334303974 & 2015 \\
\hline Amazonas & EBIRD & 525316506 & 2015 \\
\hline Amazonas & EBIRD & 525226590 & 2015 \\
\hline Amazonas & WA & 1867338 & 2015 \\
\hline Amazonas & EBIRD & 464941082 & 2016 \\
\hline Amazonas & EBIRD & 436951973 & 2016 \\
\hline Amazonas & EBIRD & 435572076 & 2016 \\
\hline Amazonas & EBIRD & 434261006 & 2016 \\
\hline Amazonas & EBIRD & 435679577 & 2016 \\
\hline Amazonas & EBIRD & 421033027 & 2016 \\
\hline Amazonas & EBIRD & 420008895 & 2016 \\
\hline Amazonas & EBIRD & 415557323 & 2016 \\
\hline Amazonas & EBIRD & 410552230 & 2016 \\
\hline Amazonas & EBIRD & 438360803 & 2016 \\
\hline Amazonas & EBIRD & 437881455 & 2016 \\
\hline Amazonas & EBIRD & 437900899 & 2016 \\
\hline Amazonas & EBIRD & 440296714 & 2016 \\
\hline Amazonas & IN & 30122542 & 2016 \\
\hline Amazonas & WA & 2596297 & 2016 \\
\hline Amazonas & EBIRD & 625625429 & 2017 \\
\hline Amazonas & EBIRD & 542606495 & 2017 \\
\hline Amazonas & EBIRD & 547687592 & 2017 \\
\hline Amazonas & EBIRD & 537918179 & 2017 \\
\hline Amazonas & EBIRD & 537611137 & 2017 \\
\hline Amazonas & EBIRD & 542657106 & 2017 \\
\hline Amazonas & EBIRD & 533018255 & 2017 \\
\hline Amazonas & EBIRD & 555661162 & 2017 \\
\hline Amazonas & EBIRD & 531133830 & 2017 \\
\hline Amazonas & EBIRD & 536713068 & 2017 \\
\hline Amazonas & EBIRD & 536104649 & 2017 \\
\hline Amazonas & EBIRD & 534090333 & 2017 \\
\hline Amazonas & EBIRD & 532965951 & 2017 \\
\hline Amazonas & EBIRD & 525715835 & 2017 \\
\hline Amazonas & EBIRD & 547819421 & 2017 \\
\hline
\end{tabular}


Apêndice.

\begin{tabular}{|c|c|c|c|}
\hline & & & \\
\hline Estado & Fonte & Tombo & Ano \\
\hline Amazonas & EBIRD & 531605851 & 2017 \\
\hline Amazonas & EBIRD & 531605715 & 2017 \\
\hline Amazonas & EBIRD & 531131988 & 2017 \\
\hline Amazonas & EBIRD & 530887556 & 2017 \\
\hline Amazonas & EBIRD & 526570581 & 2017 \\
\hline Amazonas & EBIRD & 538062167 & 2017 \\
\hline Amazonas & EBIRD & 537880449 & 2017 \\
\hline Amazonas & EBIRD & 536123011 & 2017 \\
\hline Amazonas & EBIRD & 520491270 & 2017 \\
\hline Amazonas & EBIRD & 532955653 & 2017 \\
\hline Amazonas & EBIRD & 531594082 & 2017 \\
\hline Amazonas & EBIRD & 531133681 & 2017 \\
\hline Amazonas & EBIRD & 530484714 & 2017 \\
\hline Amazonas & EBIRD & 517652299 & 2017 \\
\hline Amazonas & EBIRD & 517279798 & 2017 \\
\hline Amazonas & EBIRD & 490094077 & 2017 \\
\hline Amazonas & EBIRD & 508982109 & 2017 \\
\hline Amazonas & EBIRD & 474666154 & 2017 \\
\hline Amazonas & EBIRD & 460056070 & 2017 \\
\hline Amazonas & EBIRD & 460090617 & 2017 \\
\hline Amazonas & EBIRD & 468984196 & 2017 \\
\hline Amazonas & EBIRD & 524804304 & 2017 \\
\hline Amazonas & EBIRD & 468187882 & 2017 \\
\hline Amazonas & EBIRD & 467084256 & 2017 \\
\hline Amazonas & WA & 2833174 & 2017 \\
\hline Amazonas & EBIRD & 706730704 & 2018 \\
\hline Amazonas & EBIRD & 699279712 & 2018 \\
\hline Amazonas & EBIRD & 687907899 & 2018 \\
\hline Amazonas & EBIRD & 687159622 & 2018 \\
\hline Amazonas & EBIRD & 684548893 & 2018 \\
\hline Amazonas & EBIRD & 684226086 & 2018 \\
\hline Amazonas & EBIRD & 712178466 & 2018 \\
\hline Amazonas & EBIRD & 678050451 & 2018 \\
\hline Amazonas & EBIRD & 679171202 & 2018 \\
\hline Amazonas & EBIRD & 679007551 & 2018 \\
\hline Amazonas & EBIRD & 676748363 & 2018 \\
\hline Amazonas & EBIRD & 687907686 & 2018 \\
\hline
\end{tabular}


Apêndice.

\begin{tabular}{|c|c|c|c|}
\hline & & & \\
\hline Estado & Fonte & Tombo & Ano \\
\hline Amazonas & EBIRD & 658174483 & 2018 \\
\hline Amazonas & EBIRD & 678055031 & 2018 \\
\hline Amazonas & EBIRD & 676749681 & 2018 \\
\hline Amazonas & EBIRD & 654267718 & 2018 \\
\hline Amazonas & EBIRD & 638711978 & 2018 \\
\hline Amazonas & EBIRD & 646269622 & 2018 \\
\hline Amazonas & EBIRD & 645240103 & 2018 \\
\hline Amazonas & EBIRD & 637035161 & 2018 \\
\hline Amazonas & EBIRD & 657420677 & 2018 \\
\hline Amazonas & EBIRD & 630188372 & 2018 \\
\hline Amazonas & EBIRD & 641046258 & 2018 \\
\hline Amazonas & EBIRD & 635519894 & 2018 \\
\hline Amazonas & EBIRD & 640511063 & 2018 \\
\hline Amazonas & EBIRD & 638610938 & 2018 \\
\hline Amazonas & EBIRD & 598551956 & 2018 \\
\hline Amazonas & EBIRD & 601044161 & 2018 \\
\hline Amazonas & EBIRD & 583231710 & 2018 \\
\hline Amazonas & EBIRD & 583235100 & 2018 \\
\hline Amazonas & EBIRD & 628345934 & 2018 \\
\hline Amazonas & EBIRD & 631520525 & 2018 \\
\hline Amazonas & EBIRD & 653919081 & 2018 \\
\hline Amazonas & EBIRD & 629841170 & 2018 \\
\hline Amazonas & EBIRD & 630003335 & 2018 \\
\hline Amazonas & IN & 47189578 & 2018 \\
\hline Amazonas & IN & 45334448 & 2018 \\
\hline Amazonas & WA & 2886517 & 2018 \\
\hline Amazonas & WA & 3771481 & 2018 \\
\hline Amazonas & $x C$ & 438499 & 2018 \\
\hline Amazonas & $X C$ & 417476 & 2018 \\
\hline Amazonas & IN & 34629120 & 2019 \\
\hline Amazonas & WA & 3699579 & 2019 \\
\hline Amazonas & WA & 3367545 & 2019 \\
\hline Amazonas & WA & 3553208 & 2019 \\
\hline Amazonas & WA & 3664384 & 2019 \\
\hline Amazonas & WA & 3544364 & 2019 \\
\hline Amazonas & WA & 3647695 & 2019 \\
\hline Amazonas & WA & 3350936 & 2019 \\
\hline
\end{tabular}


Apêndice.

\begin{tabular}{|c|c|c|c|}
\hline Estado & Fonte & Tombo & Ano \\
\hline Amazonas & WA & 3468034 & 2019 \\
\hline Amazonas & WA & 3513636 & 2019 \\
\hline Amazonas & WA & 2349543 & 2019 \\
\hline Amazonas & $x C$ & 505024 & 2019 \\
\hline Amazonas & MNRJ & 7886 & \\
\hline Amazonas & MNRJ & 29472 & \\
\hline Amazonas & MZUSP & 20360 & 1939 \\
\hline Mato Grosso & EBIRD & 163564426 & 2011 \\
\hline Mato Grosso & $x C$ & 130314 & 2011 \\
\hline Mato Grosso & EBIRD & 273159119 & 2014 \\
\hline Mato Grosso & EBIRD & 269428768 & 2014 \\
\hline Mato Grosso & EBIRD & 269388867 & 2014 \\
\hline Mato Grosso & EBIRD & 273467404 & 2014 \\
\hline Mato Grosso & EBIRD & 376370980 & 2015 \\
\hline Mato Grosso & EBIRD & 422843380 & 2016 \\
\hline Mato Grosso & EBIRD & 422157464 & 2016 \\
\hline Mato Grosso & EBIRD & 416219510 & 2016 \\
\hline Mato Grosso & WA & 2103230 & 2016 \\
\hline Pará & $\mathrm{CMNH}$ & P83029 & 1921 \\
\hline Pará & $\mathrm{AMNH}$ & 285285 & 1931 \\
\hline Pará & $\mathrm{FMNH}$ & 101519 & 1937 \\
\hline Pará & $\mathrm{FMNH}$ & 101513 & 1937 \\
\hline Pará & MZUSP & 43863 & 1959 \\
\hline Pará & MZUSP & 46240 & 1961 \\
\hline Pará & MPEG & 7917 & 2001 \\
\hline Pará & MPEG & 65373 & 2008 \\
\hline Pará & WA & 839366 & 2012 \\
\hline Pará & EBIRD & 427695535 & 2013 \\
\hline Pará & EBIRD & 365477259 & 2013 \\
\hline Pará & EBIRD & 291443532 & 2013 \\
\hline Pará & WA & 1181929 & 2013 \\
\hline Pará & WA & 1340183 & 2013 \\
\hline Pará & $x C$ & 155500 & 2013 \\
\hline Pará & EBIRD & 448682683 & 2014 \\
\hline Pará & EBIRD & 282683454 & 2014 \\
\hline Pará & EBIRD & 282005306 & 2014 \\
\hline Pará & WA & 1890163 & 2015 \\
\hline
\end{tabular}


Apêndice.

(Conclusão)

\begin{tabular}{|c|c|c|c|}
\hline Estado & Fonte & Tombo & Ano \\
\hline Pará & EBIRD & 480169030 & 2017 \\
\hline Pará & EBIRD & 502810508 & 2017 \\
\hline Pará & WA & 2558990 & 2017 \\
\hline Pará & WA & 2707877 & 2017 \\
\hline Pará & WA & 2571972 & 2017 \\
\hline Pará & WA & 2777135 & 2017 \\
\hline Pará & WA & 3467598 & 2018 \\
\hline Pará & WA & 3609015 & 2019 \\
\hline Pará & ANSP & 70606 & \\
\hline Pará & MNRJ & 8523 & \\
\hline Rondônia & WA & 422050 & 2011 \\
\hline Rondônia & WA & 1048057 & 2013 \\
\hline Rondônia & WA & 2163057 & 2016 \\
\hline Rondônia & EBIRD & 567008596 & 2018 \\
\hline Roraima & WA & 48648 & 2009 \\
\hline Roraima & EBIRD & 333935163 & 2015 \\
\hline Roraima & EBIRD & 586170663 & 2018 \\
\hline Roraima & EBIRD & 590925430 & 2018 \\
\hline Roraima & WA & 3509602 & 2019 \\
\hline Roraima & WA & 3271053 & 2019 \\
\hline Roraima & WA & 3371540 & 2019 \\
\hline Roraima & WA & 3774404 & 2020 \\
\hline Roraima & WA & 3748544 & 2020 \\
\hline Roraima & MNRJ & 10097 & \\
\hline
\end{tabular}


